\title{
TRANSIENT RASH AND HAEMATURIA AFTER EXERCISE AND EMOTION
}

BY

\author{
R. S. ILLINGWORTH and K. S. HOLT \\ From the Children's Hospital, Sheffield
}

(RECEIVED FOR PUBLICATION FEBRUARY 12, 1957)

Now and again one is faced with the problem of a case of haematuria whose cause thorough investigation fails to determine. Many such cases are termed 'essential haematuria', and almost every volume of the Quarterly Cumulative Index Medicus gives one or more references to papers about this mysterious condition.

Below is an account of a child who had frequent attacks of haematuria immediately following a rash which was largely erythematous and partly urticarial, and which initially was regarded as an example of allergic haematuria.

\section{Case Report}

The patient, a girl aged $5 \frac{1}{2}$ years, was brought to the Children's Hospital, Sheffield, on account of seven attacks of haematuria in the previous four months. In all the attacks blood had been passed in single specimens only. There had been no history suggestive of nephritis, no puffiness of the eyes and no other symptoms except dysuria in some of the attacks of haematuria. She was otherwise well and full of energy.

On examination the girl was well and afebrile. She weighed $42 \mathrm{lb}$. The blood pressure was normal $(110 / 70 \mathrm{~mm}$. $\mathrm{Hg})$. There were no abnormal physical signs. The erythrocyte sedimentation rate was $13 \mathrm{~mm}$. in one hour. The blood urea was $25 \mathrm{mg}$. \%. There was a trace of albumin in the urine $(25 \mathrm{mg}$. \%), but the centrifuged deposit was normal. Nephritis was thought to be unlikely, and she was admitted for imvestigation.

Her previous and family history were irrelevant. She had been a premature baby, weighing $4 \mathrm{lb} .8 \mathrm{oz}$. Her development had been normal, and her intelligence was average. She had five siblings, and neither they nor her parents had suffered from any known allergic conditions.

On further questioning after admission the child stated that for half an hour before the first attack of haematuria she had had a measles-like rash, and that a rash had appeared before each of the six other attacks. There had also sometimes been a rash without subsequent haematuria, but never haematuria without a preceding rash. The mother added that her tongue was notably red in attacks. She always knew when haematuria was going to occur, because of the prodromal rash.

The history was suggestive of allergic haematuria, and a confident diagnosis of this condition was made after we had observed the rash.

We saw a rash (Fig. 1) on 22 occasions in her 13 weeks in hospital: on 11 of these occasions she developed naked-eye haematuria within half an hour of the appearance of the rash. The more extensive the rash, the greater was the frequency with which haematuria followed it. On two occasions she had haematuria without a preceding rash, though it is likely that a rash had occurred without being noticed. On each occasion the rash was a diffuse patchy erythema, with occasional wheals, which covered the trunk and face, and to a lesser extent the limbs. We could find no haemorrhagic element in the

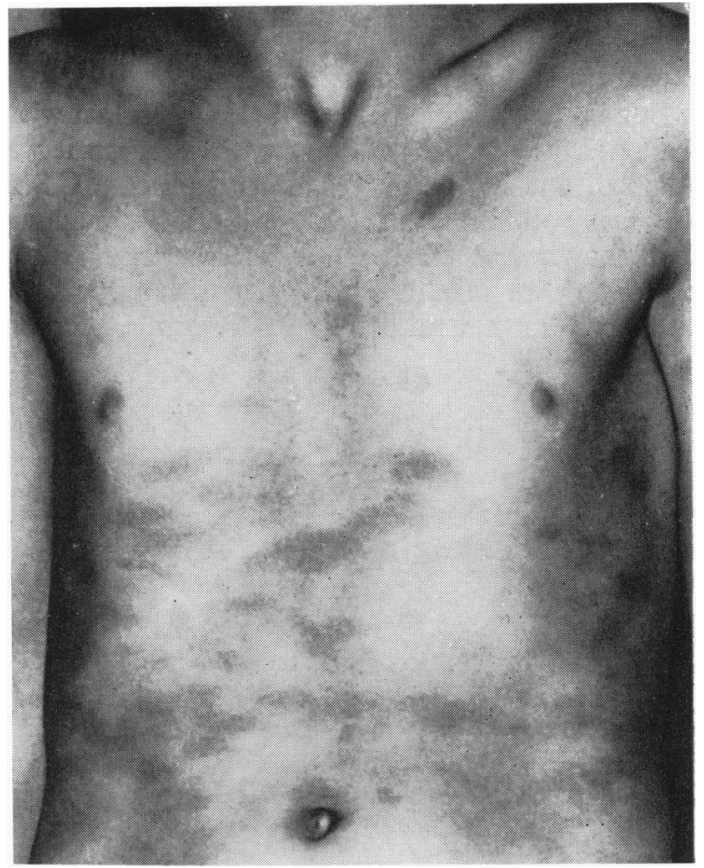

FIG. 1.-Rash immediately preceding haematuria. 
rash, even on examination with a hand lens, nor was there bleeding elsewhere, such as epistaxis or melaena.

During the attacks the blood count, including the eosinophil and platelet count, the bleeding and coagulation time and the capillary fragility tests were normal. There was no elevation of temperature in the attacks. When the haematuria occurred, there was only a small amount of albumin and there were no granular or cellular casts in the centrifuged deposit.

We were impressed by the short duration of the rash. For instance, at 9 a.m. one day the skin was clear; at 10 a.m. she developed a rash, and the tongue and palate were red, with prominent papillae: the rash lasted 30 minutes. At 10.45 a.m. there was macroscopic haematuria. At 11.15 a.m. the urine, including the centrifuged deposit, was normal.

Investigations on Admission. A throat swab grew $\beta$ haemolytic streptococci, group A.

The A.S.O. titre was 100 . Tests for C-reactive protein were negative, and mucoprotein was $1.9 \mathrm{mg}$./ $100 \mathrm{ml}$.

A Mantoux test (1 in 1,000 O.T.) was negative. Radiographs of the chest and abdomen were normal.

A blood count gave: $14 \cdot 5$ g. $\%$; R.B.C.s $4,100,000$ per c.mm.; W.B.C.s 8,000 per c.mm. (polymorphs $58 \%$, eosinophils $2 \%$, basophils $1 \%$, lymphocytes $30 \%$, monocytes $9 \%$ ); platelets 360,000 per c.mm.; bleeding time $2 \frac{1}{2}$ minutes; coagulation time 3 minutes; blood urea $35 \mathrm{mg}$. \%; the E.S.R. $9 \mathrm{~mm}$. in one hour. The Wassermann and Kahn reactions were negative. Isoagglutinins and cold agglutinins were absent.

Serum proteins totalled 6.5 g. $\%$ (albumin 3.8 g. $\%$ and globulin $2.7 \mathrm{~g}$. \%). Electrophoresis showed some increase in $\alpha_{2}$ and $\beta$ globulin, but no cryoglobulin.

Skin tests for common allergens were negative.

Spectroscopic examinations of urine in attacks showed no haemoglobinuria.

Shortly after her admission we took her to the theatre for cystoscopy immediately after she had passed a grossly bloodstained specimen of urine, only to find that the urine had returned to normal. As mentioned below, this rapid clearing of the haematuria may have been due to premedication with atropine. Further cystoscopy was not thought to be justified and for the same reason sigmoidoscopy and proctoscopy were not carried out.

We began a series of elimination diets, but made no progress in elucidating the cause, for attacks continued to occur irrespective of the diet which she was having.

It was then realized that there was an emotional factor in the attacks, for on at least three occasions the rash developed immediately after a venipuncture, and on three or more occasions during the professorial ward round. Her mother then said that over-excitement was a factor in the production of the rash, for she had noticed that it had preceded at least three of the attacks.

After three months' observation and study, we felt that we were making no progress in the elucidation of the problem, and once more discussed the matter with her mother who then remarked that she was sure that she knew why the girl had haematuria: it was caused, she said, either by over-excitement or over-exertion. We then tried the effect of exertion and promptly precipitated an attack. This was repeatedly confirmed, and at a clinical meeting of the Sheffield Regional Paediatric Society we showed the girl without a rash and with normal urine, and then caused two medical students to exercise her in the adjacent park. She returned 20 minutes later with a profuse erythematous rash and gross macroscopic haematuria.

\section{Discussion}

At first it seemed likely that this child was suffering from allergic haematuria. Haematuria has been known to follow injections of T.A.B. (Coca, 1930), tetanus antitoxin (Rhodes, 1937), dust extract (Adelsberger, 1931), insulin (Henderson, 1927), the ingestion of codfish (Miller and Uhle, 1939), milk (Kittredge and Johnson, 1949), goat milk (Blaustein, 1926), potassium iodide (Withers, 1956) and the inhalation of allergens (Thomas and Wicksten, 1944). The subject is reviewed by Urbach and Gottlieb (1946). Subsequently we discarded the diagnosis of allergic haematuria.

It was realized that the rash was probably identical with urticaria of nervous origin described by Grant, Pearson and Comeau (1936). Our patient's rash was similar to that described by these authors in its distribution, appearance, precipitation by exercise and emotion, and its transience. Warmth also precipitated the rash but it was a less powerful stimulant than either exercise or emotion. The rapid disappearance of the rash and haematuria just before cystoscopy may have been due to premedication with atropine (gr. $1 / 100,0.65 \mathrm{mg}$.). We tried the effect of giving atropine (gr. 1/150) just before exercise, and the resulting rash was not followed by haematuria. Atropine did not abolish the attack as in the case reported by Grant et al. (1936) but modified the attack as in the case reported by Herxheimer (1956). We applied a $10 \%$ aqueous solution of atropine sulphate to the skin, and found that it abolished the rash in that area just as it did in the case reported by Herxheimer (1956).

It appears, therefore, that the rash observed in our patient is almost certainly identical with urticaria of nervous origin. The association of haematuria with this condition is not mentioned by Herxheimer (1956) or Morgan (1953), nor could we find any paper referring to it in a search through the Quarterly Cumulative Index Medicus. One of the original writers to describe urticaria of nervous origin is unaware of any connexion between this condition and haematuria (Grant, 1956). Transient haematuria can occur apparently after exercise. Boone, Haltiwanger and Chambers (1955) described football haematuria. Six out of 37 football players showed gross but transient haematuria after a strenuous 
game. No mention is made, however, whether any of these players had a rash.

\section{Summary}

We have described a 5-year-old child who had repeated attacks of a rash followed frequently by haematuria. These attacks immediately followed emotion or exercise.

\section{REFERENCES}

Adelsberger, L. (1931). Dtsch. med. Wschr., 57, 585. Blaustein, N. (1926). J. Urol. (Baltimore), 16, 379.
Boone, A. W., Haltiwanger, E. and Chambers, R. L. (1955). J. Amer. med. Ass., 158, 1516.

Coca, A. F. (1930). Bull. N.Y. Acad. Med., 6, 593.

Grant, R. T. (1956). Personal Communication.

-, Pearson, R. S. B. and Comeau, W. J. (1936). Clin. Sci., 2, 253.

Henderson, J. (1927). Brit. med. J., 1, 231.

Herxheimer, A. (1956). Clin. Sci., 15, 195. Kittredge, W. E. and Johnson, C. (1949). New Orleans med. Surg. J., $101,419$.

Miller, M. W. and Uhle, C. A. W. (1939). Int. Clin. n.s. 2, 3, 183.

Morgan, J. K. (1953). J. invest. Dermat., 21, 173.

Rhodes, J. (1937). J. Urol (Baltimore), $38,410$.

Thomas, J. W. and Wicksten, V. P. (1944). Ann. Allergy, 2, 396

Urbach, E. and Gottlieb, P. M. (1946). Allergy, 2nd ed. New York. Withers, O. R. (1956). In Current Therapy, 1956, ed. by Conn, H. F. Philadelphia. 